\title{
Budget impact analysis in economic evaluation: a proposal for a clearer definition
}

\author{
Livio Garattini $\cdot$ Katelijne van de Vooren
}

Published online: 27 August 2011

(C) Springer-Verlag 2011

Budget impact analysis (BIA) is a relatively recent method for economic evaluation (EE) in the field of health care. It assesses the financial consequences of the introduction of a new technology in a specific setting in the short-to-medium term [1]. BIA is supposed to be complementary to more established types of EEs, providing additional information for decisions on the reimbursement of new technology [1].

Several countries (e.g. Australia, Canada, Belgium, Croatia, Hungary, and Poland) require manufacturers to make a BIA at present, often alongside a cost-effectiveness analysis (CEA), to support applications for national or regional reimbursement [2]. Two main reasons are often mentioned for healthcare systems' increasing interest [3, 4] in this technique. First, they have to deal with continuous development of technology on one hand, and budget constraints on the other. While CEA-broadly including costutility analysis (CUA) [5] - may help assigning priority to interventions, BIA is perceived as useful in assessing their sustainability, a major concern for budget holders with scarce financial resources.

A second reason for interest in BIA seems to be the lack of responsiveness and the complexity of CEA to the needs of budget holders and decision makers in health care. According to best practice [6], CEA should take a societal perspective and a long enough time horizon to include all the benefits of a new technology, and this often implies

\footnotetext{
L. Garattini $(\bowtie) \cdot$ K. van de Vooren

Department of Public Health, 'Mario Negri' Institute for Pharmacological Research, CESAV, Centre for Health

Economics, Via Camozzi, 3 c/o Villa Camozzi, 24020 Ranica, Bergamo, Italy

e-mail: livio.garattini@marionegri.it

K. van de Vooren

e-mail: katelijne.vandevooren@marionegri.it
}

modeling for life-time estimates. A societal perspective does not fulfill healthcare decision makers' expectations since they can only manage their own budget, and potential savings due to reduction in productivity losses, for instance, fall outside their span of control. Even a CEA limited to the healthcare system's perspective does not necessarily overcome this concern, since the 'silos effect' (i.e. the difficulty of redeploying costs and savings from one budget to another) persists in healthcare management too.

A long-term horizon still raises the question whether to start spending immediately on a new treatment that might lead to savings in the future. This sort of measure can only be introduced by additional funding or by disinvesting from alternative treatments, the latter generally being very difficult in practice [1].

To summarize, these issues show the limitations of CEA from the budget holder's viewpoint. BIA should provide useful information to tackle the new hurdle of "affordability", after having fulfilled the better-known hurdles of "safety", "efficacy", and "added value" of new treatments over existing ones [1, 3, 7].

To our knowledge, Trueman et al. [1] were the first to introduce BIA in the healthcare literature, in 2001. They roughly defined BIA as an analysis that 'falls somewhere between a simple 1-year accounting model and the costing side of an economic evaluation from a societal point of view'. We could not find a specific definition of BIA in Drummond et al. [6], the most acknowledged and widely used manual on EE in healthcare worldwide (translated into several languages).

We reviewed the international literature focusing on BIA methodology to see whether the first wide-range definition had been elaborated further to better clarify BIA's role in relation to the other types of EE. 


\section{Literature search}

A literature search was done on the international database PubMed to find all the articles on BIA methodology published in English in the last decade (January 2001-May 2011). Figure 1 summarizes the search strategy and its results. Only five articles [1-3, 7, 8] that discussed BIA methods specifically were reviewed according to a list of key points derived from our proposal for Italian pharmacoeconomic guidelines [9]: study perspective, alternatives, time horizon and discounting, costs, effectiveness, results and sensitivity analysis. To make this exercise as useful as possible for budget holders, we tried to highlight the main differences between CEA and BIA, the former being by far the most widely used EE in health care $[10,11]$.

\section{Study perspective}

The BIA perspective should be restricted to healthcare services, unlike what is recommended for CEA, i.e. to take a perspective that assesses the value of a new technology for society as a whole [6]. Only using this narrower viewpoint can BIA be expected to respond to budget holders' needs. Although the range of interest of each budget holder can vary according to the role (e.g. the Canadian guidelines limit it to the pharmaceutical budget [2]), all the articles reviewed [1-3, 7, 8] agreed on this basic statement, as expected.

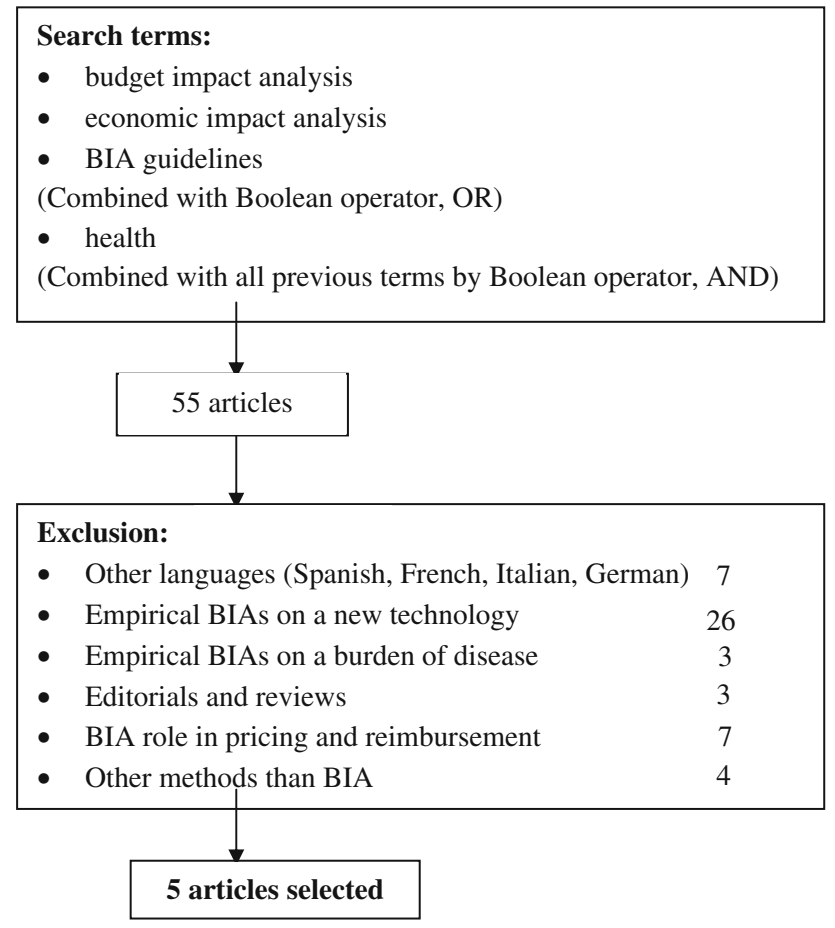

BIA, budget impact analysis

Fig. 1 Literature search strategy. BIA budget impact analysis
Another difference between CEA and BIA is the possibility of using virtual populations in a CEA (e.g. theoretical cohorts in Markov models), while BIA should be restricted to real populations in national or local settings, in line with the perspective chosen.

\section{Alternatives}

Whereas in a CEA one or more technologies can be compared to 'do nothing' and/or between themselves, a BIA is expected to compare two scenarios only. The reference scenario comprises the current treatment mix of the healthcare setting analyzed, while the alternative scenario estimates the impact of the new technology. All the articles reviewed stressed that the new scenario should take account of market dynamics, particularly the uptake and substitution rates of the new technology.

\section{Time horizon and discounting}

The time horizon (TH) in a CEA should be long enough to include all the benefits of the new technology. To be responsive to budget holders' needs, all authors agree that the TH should be limited to the short-to-medium term in a BIA. Five years was the longest $\mathrm{TH}$ in the articles reviewed.

Discounting is necessary for medium-to-long-term studies [6] and almost all five articles shared the recommendation not to apply discounting in a BIA. This was justified not only by BIA's shorter TH, but also by the budget holder's interest in real financial streams over time. Only the Polish guidelines [7] suggested applying a yearly discount rate of $5 \%$ to studies adopting a $\mathrm{TH}$ longer than 2 years.

Costs

Of course, the types of costs in an EE depend on the perspective of the analysis. This implies that only healthcare costs should be taken into account in a BIA, while a CEA can include patients' direct costs and indirect costs too.

Four articles $[2,3,7,8]$ in our review agreed that only the actual payments made or savings achieved by the budget holder should be considered, privileging prices, charges and tariffs as unit costs, and actual usage in the setting analyzed as resource consumption. Trueman et al. [1] were less specific on how to assess costs, only claiming these had to be consistent with the budget holder's perspective.

\section{Effectiveness}

Effectiveness in full EEs can be incorporated using various measures, e.g. life years gained (LYGs) in a CEA and 
quality-adjusted life years (QALYs) in a CUA. All the articles we reviewed agreed that effectiveness was part of the overall assessment of a new technology, but stated much less clearly whether and how to include it in a BIA. ISPOR guidelines [2], in the recommendations for inputs and data sources, pointed out that efficacy and safety are generalizable aspects of interventions, in contrast to the other data inputs which have to be local, but gave no further details on how to incorporate efficacy. Nuijten et al. [8] claimed that specific information on the prognosis of the disease was needed to decide on the type of model to apply in a BIA, its characteristics and effectiveness measure(s), although they provided no specific explanation in this case too.

Results

CEA results are reached by calculating the incremental cost-effectiveness ratio (ICER), i.e. using incremental analysis to quantify the extra cost of obtaining an additional unit of outcome [6]. Results in a BIA do not seem to require specific calculations on the outcomes of existing technologies and the new one. Both the total budgetary impact of the new scenario and its incremental impact (against the reference scenario) should be calculated according to the Canadian [3] and ISPOR [4] guidelines. The Polish guidelines [7] recommend including the impact on the whole healthcare service in the results. To address the budget holder's needs, most authors [1, 3, 7] agreed that results should be split in their presentation into natural and financial units, and periodic budget rounds too.

\section{Sensitivity analysis}

A sensitivity analysis (SA) is recommended to test the robustness of results in any type of EE [6]. Several forms can be used in a CEA (one-way, multi-way, analysis of the extremes, probabilistic), depending also on the extensive recourse to modeling. All studies agreed that BIA, which needs forecasts on the uptake of the new technology, is prone to considerable uncertainty. Standard SA is considered inadequate, because of the lack of appropriate data distribution [8]. Scenario analysis should be a useful addition to traditional SA methods depending on the budget holder's needs [2, 8]. The two national guidelines reviewed listed specific requirements for parameters to be subjected to SA. For instance, Polish guidelines [7] recommended that inputs such as diffusion rate and probability of redeploying resources should be varied in any model, while Canadian guidelines [3] required at least tests of market share estimates and unknown prices for any drug likely to obtain listing imminently.

\section{From theory to practice}

To assess what methods were applied in studies in real practice, we examined the recent review by Orlewska et al. [4] describing how published BIAs adhere to ISPOR guidelines [2]. The general agreement in the articles on BIA methodology on the need to take the budget holder's perspective was mirrored in practice, all empirical BIAs having taken this view. There was also consistency between theory and practice on the alternatives to be compared (the present scenario and the future one including the new technology), the types of costs to be included (i.e. healthcare costs only), and the presentation of results (i.e. total costs of both alternatives) in a BIA. Despite full agreement on the need for SA, only three-quarters of the BIAs (76\%) actually did it, mostly in the form of alternative scenarios or univariate analyses. Whereas the literature on BIA recommends a short/medium-term $\mathrm{TH}$, a small proportion of studies (16\%) considered a TH longer than 5 years. Thus, some discrepancy was found in discounting too, which was applied in half of the studies with a TH longer than 1 year.

Although Orlewska et al. did not say whether effectiveness was included, we concluded from their synoptic table that $35 \%$ of the studies somehow took account of the effectiveness of the new technology. To assess further the inclusion of effectiveness in published BIAs, we reviewed the abstracts of the 26 studies retrieved in our literature search (Fig. 1). Seventeen (65\%) discussed effectiveness, monetized as savings from other healthcare resources, confirming our concern about the lack of clarity on this crucial issue in BIA methodology.

\section{Proposal}

In the absence of an exhaustive definition for BIA in literature and in the light of growing demand from budget holders in many countries, we tried to provide a 'stand alone' definition (Table 1), which extends the still complementary present one. According to this proposal, BIA should be defined as an EE conducted: (1) according to the budget holders' perspective; (2) with a short TH ( $\leq 3$ years) and within a clearly specified setting; (3) where results are expressed as undiscounted cost differences between the new scenario (including the new technology) and the current/reference scenario; (4) taking account of the potential trade-offs in healthcare resources induced by the effectiveness of the new technology, and (5) examining the results using SA responsive to the uncertainty surrounding future market developments (like scenario analysis), and easy to understand by budget holders (like the analysis of extremes). 
Table 1 Proposal for a definition of BIA compared to CEA

\begin{tabular}{lll}
\hline & BIA & CEA \\
\hline Study perspective & Budget holder & Variable (societal, healthcare system, patient) \\
Alternatives & Current treatment mix & One or more technologies \\
Time horizon & Short $\leq 3$ years & Variable (short, medium, long) \\
$\begin{array}{l}\text { Discounting } \\
\text { Costs }\end{array}$ & Unnecessary & To apply to studies with medium/long-term time horizons \\
& Direct healthcare costs & Direct (health care and non-health care) and indirect costs, \\
Effectiveness & depending on the study perspective \\
Results & Short-term costs and savings in healthcare services & Natural units (e.g. LYG) and quality of life* (e.g. QALY) \\
Sensitivity analysis & Ancremental healthcare costs & Incremental cost-effectiveness ratio \\
& & Various forms of sensitivity analysis (one-way, multi-way, \\
& & extremes, scenarios, probabilistic, threshold)
\end{tabular}

$B I A$ budget impact analysis, $C E A$ cost-effectiveness analysis, $L Y G$ life year gained, $Q A L Y$ quality-adjusted life year

* Cost-utility analysis

In our proposal for a complete BIA definition, perspective, alternatives and costs fully reflect the recommendations in the articles reviewed. We share with most of the articles the indications on $\mathrm{TH}$, discounting and $\mathrm{SA}$, but try to be more conservative by recommending an upper limit of 3 years as TH, excluding discounting and limiting SA to extremes and scenario analyses. The underlying justification is that such choices should be more consistent with budget holders' interests to produce BIAs expressed in real financial streams, with easy-to-understand estimated results.

The most important novelty in our proposal is a clear statement on whether and how to include effectiveness in the results, i.e. as potential short-term savings in healthcare costs induced by innovative technologies. Although costbenefit analysis (CBA) translates effectiveness in monetary terms too, the underlying process is different. In a CBA benefits should be evaluated according to people's observed or stated preferences (usually based on the willingness-to-pay approach), while in a BIA benefits should result only from savings in healthcare resources induced by the new technology.

Including effectiveness in a BIA can facilitate the budget holder's decision on reimbursement since certain technologies might show that their introduction could be less costly in the short-term thanks to trade-offs on other healthcare resources. For instance, this may be the case for drugs/medical devices/surgical procedures in hospitals that can reduce the days of stay and/or diagnostic tests. On the other hand, this cannot happen by definition for technologies that are unable either to translate effectiveness into savings (like anti-cancer drugs prolonging survival) or to show short-term savings (e.g. chronic drugs and vaccinations). A simple forecast of future expenses induced by the adoption of the new technology should be the only reasonable BIA worth doing in such circumstances.

In conclusion, once the inclusion of short-term consequences on healthcare costs induced by effectiveness has been agreed, BIA could find a more specific place among EEs available in health care. Consequently, it could be embedded more homogeneously in the regulatory requirements of public authorities that decide to adopt it.

\section{References}

1. Trueman, P., Drummond, M., Hutton, J.: Developing guidance for budget impact analysis. Pharmacoeconomics 19, 609-621 (2001)

2. Mauskopf, J.A., Sullivan, S.D., Annemans, L., et al.: Principles of good practice for budget impact analysis: report of the ISPOR task force on good research practices-budget impact analysis. Value Health 10, 336-347 (2007)

3. Marshall, D.A., Douglas, P.R., Drummond, M.F., et al.: Guidelines for conducting pharmaceutical budget impact analyses for submission to public drug plans in Canada. Pharmacoeconomics 26, 477-495 (2008)

4. Orlewska, E., Gulácsi, L.: Budget-impact analyses, a critical review of published studies. Pharmacoeconomics 27, 807-827 (2009)

5. Weinstein, M., Siegel, J., Gold, M., Kamlet, M., et al.: Recommendations of the panel on cost-effectiveness in health and medicine. JAMA 276, 1253-1258 (1996)

6. Drummond, M., O’Brien, B., Stoddart, G., Torrance, G.: Methods for the economic evaluation of health care programmes, 2 nd edn. Oxford Medical Publications, Oxford (1997)

7. Orlewska, E., Mierzejewski, P.: Proposal of polish guidelines for conducting financial analysis and their comparison to existing guidance on budget impact in other countries. Value Health $\mathbf{7}$, $1-10(2004)$

8. Nuijten, M., Mittendorf, T., Persson, U.: Practical issues in handling data input and uncertainty in a budget impact analysis. Eur. J. Health Econ. 12, 231-241 (2011)

9. Garattini, L., Grilli, R., Scopelliti, D., Mantovani, L.: A proposal for Italian guidelines in pharmacoeconomics. Pharmacoeconomics 7, 1-6 (1995)

10. Drummond, M., Dubois, D., Garattini, L., Horisberger, B., et al.: Current trends in the use of pharmacoeconomics and outcomes research in Europe. Value Health 2, 323-332 (1999)

11. Hutter, F., Antoñanzas, F.: Economic evaluations in the EURONHEED: a comparative analysis. Pharmacoeconomics 27, 561-570 (2009) 